\title{
IMPLEMENTAÇÃO DO SISTEMA DE AUTOMAÇÃO DA QUARTA USINA DE PELOTIZAÇÃO DA SAMARCO - PROJETO P4P, UTILIZANDO O MODELO DE FORNECIMENTO DO TIPO MAC - MAIN AUTOMATION CONTRACTOR*
}

\author{
Paulo Correa \\ Willians Rodrigues da Silva ${ }^{2}$
}

\section{Resumo}

No processo de implementação de uma nova planta industrial, a disciplina de automação, responde pela integração dos equipamentos de forma a permitir a operação segura e produtiva dos dispositivos envolvidos, com um mínimo de intervenção humana. Em projetos de grande porte, temos muitos fornecedores de equipamentos mecânicos com diversas formas de contratação e estabelecer um padrão único para o sistema de automação, tornase uma atividade complexa, porém, fundamental para o sucesso do projeto. Por outro lado, o sistema de controle é sempre o último a ser montado e pode provocar atrasos no comissionamento dos equipamentos, se não estiver devidamente pronto, ou mesmo ser pressionado para compensar atrasos ocorridos nas etapas anteriores da construção. Nesse contexto, a engenharia de automação precisa estar concluída, os materiais precisam estar disponíveis e a mão de obra pronta para atendimento no exato momento das necessidades. Mediante o uso de uma estratégia de contratação antecipada do pacote de automação, na modalidade MAC, onde apenas uma empresa responde pelo fornecimento global do sistema de automação e instrumentação, reduzindo as interfaces envolvidas, buscando a participação do fornecedor nas fases iniciais da engenharia e com a elaboração de um planejamento consistente de recursos, foi possível atender as demandas da construção e comissionamento, dentro do cronograma e custo previstos para o projeto. Os resultados alcançados pela disciplina de automação e controle, aliados ao resultado global do projeto, sinalizam a assertividade das ações realizadas, trazendo benefícios consideráveis e reduzindo o risco na implementação do projeto $\mathrm{P} 4 \mathrm{P}$.

Palavras-chave: Projeto P4P; Samarco; Automação; MAC.

\section{IMPLEMENTATION OF AUTOMATION SYSTEM FOR SAMARCO FOURTH PELLET PLANT - P4P PROJECT, USING MAC MODEL - MAIN CONTRACTOR AUTOMATION} \section{Abstract}

In the process of implementation of a new industrial plant, automation discipline, is responsible for the integration of the equipment in order to allow productive and safe operation of the devices involved, with a minimum of human intervention. In large projects, we have many suppliers of mechanical equipments with various forms of contracting and establish a single standard for the automation system, it becomes a complex activity, however, critical to the success of the project. On the other hand, the system of control is always the last one to be mounted and may cause delays in the commissioning of the equipment, if it is not properly ready, or even being pressured to compensate for delays occurring in the previous steps of the construction. In this context, automation engineering needs to be completed, the materials must be available and ready workforce for attendance at the exact moment of the needs. Through the use of a strategy of hiring automation package, advance in MAC mode, where only one company is responsible for the global supply of the automation system and instrumentation, reducing the interfaces involved, seeking the participation of supplier in the early stages of engineering and with the development of a consistent planning of resources, it was possible to meet the demands of construction and commissioning, on schedule and cost for the project. The results achieved by the automation and control discipline, allies to overall result of the project, signaling the assertiveness of the actions carried out, bringing considerable benefits and reducing risk in the implementation of $\mathrm{P} 4 \mathrm{P}$ project.

Keywords: Project P4P; Samarco; Automation; MAC.

Engenheiro Eletricista, pós-graduado em Automação e Controle de Processos Industriais, Especialista de Automação, lotado na Gerência Geral de Engenharia da Samarco Mineração S/A, Anchieta, ES, Brasil.

2 Engenheiro, Especialista de Automação, lotado na Gerência Geral de Engenharia da Samarco Mineração S/A, Mariana, MG, Brasil. 


\section{INTRODUÇÃO}

Fundada em 1977, a Samarco é uma empresa brasileira de mineração, de capital fechado, controlada em partes iguais por dois acionistas: BHP Billiton Brasil Ltda. e Vale S.A. Nosso principal produto são pelotas de minério de ferro, produzidas a partir da transformação de minerais de baixo teor em um produto nobre, de alto valor agregado, e comercializado para a indústria siderúrgica mundial.

Em abril de 2014, a Samarco comemorou mais um marco histórico do seu crescimento: a entrega da Quarta Usina de Pelotização.

O projeto de expansão movimentou as duas unidades da Samarco: em Germano (MG), foi construído um terceiro Concentrador, com capacidade de produção de 9,5 milhões de toneladas anuais; em Ubu (ES), a empresa levantou sua quarta Usina de Pelotização, capaz de produzir 8,25 milhões de toneladas anuais.

Interligando as duas pontas, foi construído o terceiro Mineroduto da empresa, paralelamente aos outros dois já existentes. Com 400 quilômetros de extensão, ele passa por 25 municípios, mineiros e capixabas, e tem capacidade para transportar 20 milhões de toneladas por ano.

O projeto da Quarta Usina de Pelotização, chamado de projeto P4P, possibilitou o aumento da capacidade produtiva da empresa em $37 \%$, elevando a capacidade de produção de pelotas de minério de ferro de 22,25 milhões de toneladas ao ano para 30,5 milhões.

Não se trata apenas de uma obra com investimentos de $R \$ 6,4$ bilhões, essa é uma iniciativa com impactos positivos, que traz para o Brasil o maior forno de pelotização já construído, na unidade de Ubu, com $816 \mathrm{~m}^{2}$ de área de grelha, e contribui para que o país seja referência em capacidade e competência no setor de mineração.

Os números abaixo representam o tamanho do projeto:

- O tempo de duração das obras foi de 35 meses.

- No pico da obra, 13 mil empregos foram gerados e, 1100 novos empregos diretos e indiretos foram criados.

- Mais de 63,1 milhões de homens-horas trabalhadas sem a ocorrência de acidente fatal ou incapacitante.

$\mathrm{Na}$ sequência estaremos descrevendo as etapas envolvidas na engenharia, aquisição, construção e comissionamento do sistema de automação e controle, objeto desse trabalho.

\section{PREMISSAS PARA O SISTEMA DE AUTOMAÇÃO}

Durante o ano de 2003, realizamos um estudo para avaliar as tendências tecnológicas para os sistemas de automação. O levantamento incluiu visitas aos fornecedores, visitas aos usuários e testes em bancada para avaliação dos sistemas. Baseado nessas informações, estabelecemos os critérios básicos para o fornecimento do Sistema de Automação do Projeto da Terceira Usina de pelotização da Samarco, projeto P3P.

No inicio de 2008, a terceira usina entrou em operação e com base nos resultados alcançados, com ganhos significativos com relação ao custo da montagem e tempo de comissionamento, ratificaram que as premissas adotadas no desenvolvimento do projeto P3P estavam alinhadas com as tendências tecnológicas e com as necessidades da empresa. 
Para o projeto $\mathrm{P} 4 \mathrm{P}$, foram mantidas as mesmas premissas do projeto $\mathrm{P} 3 \mathrm{P}$, ou seja, utilização o conceito de controladores híbridos com interligação dos sinais de campo, através de redes industriais, conforme abaixo:

- Sinais digitais e analógicos $(4 \sim 20 \mathrm{~mA})$, conectados em painel de remotas interligados em rede profibus DP;

- Sinais de instrumentação inteligente, conectados através de redes Foundation Fieldbus;

- Centro de controle de motores, conectados através de redes Profibus DP;

- Dispositivos com controle dedicado, por exemplo; balanças e compressores, são conectados através de interface de comunicação profibus DP.

A arquitetura do sistema se manteve, porém foram identificadas algumas oportunidades de melhorias, relatadas durante a fase de lições aprendidas do projeto P3P e que iremos detalhar no próximo item, como um dos desafios na implementação do novo projeto de automação.

Descrever brevemente os equipamentos e os procedimentos utilizados, assim como a literatura e os métodos estatísticos empregados, quando for o caso.

\section{DESAFIOS NA IMPLEMENTAÇÃO DO PROJETO DE AUTOMAÇÃO}

\subsection{Desafio 1 - Lições Aprendidas - Projeto P3P}

Apesar do sucesso alcançado no projeto $\mathrm{P} 3 \mathrm{P}$, tivemos vários problemas na implementação do projeto decorrentes dos seguintes fatos:

- A documentação gerada pela empresa projetista, não permitia a configuração do sistema.

- Os típicos para configuração do sistema, não foram previamente definidos.

- Durante a fase de configuração, tivemos muito retrabalho para adequação do programa aplicativo.

- Alguns controladores não suportaram a carga e tiveram que ser duplicados.

- O modelo de contratação do pacote de automação ficou restrito ao fornecimento do hardware, software, programa aplicativo e algumas horas de comissionamento.

- A contratação do fornecedor do sistema de automação foi feita na final da fase de engenharia.

- Na montagem das redes não foram observadas as boas práticas de engenharia, resultando em problemas de conectorização, aterramento e passagem de cabos de redes em conjunto com cabos de força.

- A compra dos instrumentos foi feita por tipo de instrumento, sendo necessária a abertura de diversos processos de aquisição, análises técnicas e comerciais, resultando em diversos fornecedores.

- Alguns instrumentos foram especificados inadequadamente pela empresa de engenharia projetista.

Em consequência tivemos um aumento do custo do projeto P3P, devido principalmente a necessidade de contratação de horas adicionais de engenharia.

Para o próximo projeto, o grande desafio seria encontrar soluções para os problemas acima citados, não poderíamos cometer os mesmos erros. 


\subsection{Desafio 2 - Equipamentos Fornecidos em Regime EPC}

Em um projeto de grande porte, que envolve grande quantidade de equipamentos, soluções de aquisição do tipo turn-key, onde o fornecedor entrega o equipamento funcionando, são inevitáveis. Para o sistema de automação, esse tipo de fornecimento pode representar um problema no futuro, se a engenharia não agir no sentido de padronizar o fornecimento dos equipamentos, o sistema de automação pode se transformar em uma colcha de retalhos, com vários subsistemas de automação, disponibilizados pela área industrial, com consequências críticas na manutenção e operação da planta.

Esse aspecto é determinante para que a escolha do fornecedor do sistema de automação seja priorizada nos processos de aquisição, antes mesmo das definições dos fornecedores dos equipamentos mecânicos.

Somente com a definição prévia do fornecedor do pacote de automação, podemos negociar o fornecimento do equipamento mecânico no padrão requerido pelo sistema de automação e encontrar soluções para as exceções justificadas e para isso a presença da engenharia do fornecedor do sistema de automação é essencial.

\subsection{Desafio 3 - Novo Projeto P4P, Utilizando Expansão de Áreas Existentes em Conjunto com as Áreas Totalmente Novas.}

$\mathrm{Na}$ fase de estudo conceitual, o P4P estava projetado para ter somente áreas novas, sem qualquer interferência com a atual operação das plantas existentes. Este conceito era muito bom para a construtibilidade da nova planta, porém essa possibilidade tornava o projeto inviável economicamente.

A solução encontrada para viabilizar o projeto, foi repotenciar algumas áreas e equipamentos existentes, em conjunto com novas áreas, de forma a atender as necessidades do novo projeto e mantendo a capacidade das plantas em operação.

Do ponto de vista de automação, essa solução contribuiu para a definição do fornecedor do sistema de automação, visto não ter sentido investir na aquisição de um novo sistema para controlar equipamentos instalados na mesma área de processo.

Em contrapartida, haveria a necessidade de realizar estudos no sistema atual, para verificar se esse aumento na área expandida poderia ser absorvido e definir a arquitetura do sistema com essa nova concepção.

\subsection{Desafio 4 - Aplicação de Novas Tecnologias}

Apesar da manutenção das premissas básicas em relação ao projeto anterior, não podemos esquecer, que os sistemas de automação estão em constante evolução. Portanto a engenharia de automação precisar estar atenta às novas tecnologias e empregá-las para obter ganhos, não somente na redução de custo e prazo, mas também visando melhorias e otimizações futuras nos processos industriais.

\section{BUSCANDO SOLUÇÕES PARA O SISTEMA DE AUTOMAÇÃO}

Além dos desafios apresentados no tópico anterior, o novo projeto foi aprovado com um valor de Capex e prazo, extremamente arrojados para a dimensão do projeto. 
Portanto o cumprimento de prazos e custos são também desafios para os quais possíveis desvios possuem pouca tolerância.

Em meio a essa situação, a engenharia de automação definiu algumas necessidades para a contratação do pacote de automação:

- Antecipar a contratação do pacote de automação, de forma a termos a participação do fornecedor nas definições de engenharia do sistema de automação;

- Aumentar o escopo de fornecimento incluindo os instrumentos do sistema de controle;

- Incluir o fornecimento de serviços especializados, tais como: supervisão de montagem das redes industriais, conectorização das redes, supervisão de montagem de instrumentos, analise e supervisão do sistema de aterramento, entre outros.

A solução encontrada para atender as necessidades do projeto evoluiu para o conceito de MAC - Main Automation Contractor, ou seja:

"Estabelecer um escopo de fornecimento para uma solução completa de automação e instrumentação, de forma a garantir um padrão único para o projeto, com a presença de especialistas, reduzindo o número de empresas envolvidas, buscando sistemas modernos e inovadores, dentro do prazo e custo compatíveis com o projeto."

\section{ESCOPO DETALHADO DO FORNECIMENTO MAC}

No escopo detalhado foram incluídos, os seguintes itens:

- Todos os equipamentos do sistema de automação, incluindo os computadores, controladores, sistemas UPS, painéis de remotas de campo, painéis de válvulas, painéis de relé de segurança, quadros de distribuição de tensão e transformadores de controle.

- Todos os instrumentos, especificados pela projetista. Os instrumentos fornecidos, junto com os equipamentos mecânicos, não fizeram parte do fornecimento MAC.

E os seguintes serviços:

- Gerenciamento do Projeto

- Analise da documentação de engenharia

- Definição dos padrões de configuração - Function Designer System - FDS

- Desenvolvimento do aplicativo do Sistema de Controle e Supervisão

- Testes de integração com dispositivos profibus DP e instrumentos Fieldbus Foundation - FF

- TAF - Teste Aceitação da Configuração

- Serviços de Comissionamento e operação assistida;

- Serviços de Treinamento de Configuração, Operação e Manutenção;

- Serviços de documentação e "As Built" do sistema de controle;

- Sintonia de Parâmetros de Controladores PID;

- Integração com o PIMS Samarco;

- Racionalização de Alarmes, conforme norma ISA S18.2, EEMUA 191, 192);

- Consolidação das folhas de dados de instrumentos;

- Definição dos típicos de montagem de instrumentos;

- Supervisão de montagem dos instrumentos;

- Conectorização dos cabos de redes FF e DP; 
- Certificação das redes industriais;

- Interligação dos instrumentos no barramento FF;

- Configuração dos instrumentos e integração no sistema de gerenciamento de ativos;

- Analise do projeto de aterramento e acompanhamento de montagem;

- Gerenciamento de entrega e acondicionamento dos instrumentos no almoxarifado da obra.

\section{ESCOLHA DO FORNECEDOR DO SISTEMA DE AUTOMAÇÃO}

Uma vez definido o modelo de contratação, a etapa seguinte foi à negociação para a escolha do fornecedor do pacote de automação, que deixou de ser apenas um fornecedor de hardware e software, passando a ser o fornecedor da solução completa de automação e controle, incluindo serviços especializados de montagem e engenharia.

$\mathrm{Na}$ escolha do fornecedor, os requisitos de fornecimento aplicados no processo de aquisição, resultaram na aplicação do conceito de fornecedor único, pois como estamos trabalhando com expansões de áreas existentes, não tinha sentido trabalhar com outro fornecedor senão o mesmo do projeto anterior.

\section{FATORES CRÍTICOS PARA O SUCESSO}

Vencida a etapa de contratação antecipada do pacote de automação e controle, com o escopo abrangente de fornecimento, a engenharia de automação do projeto, elaborou em conjunto com o fornecedor, uma relação de atividades classificadas como críticas para o sucesso o empreendimento.

O controle eficiente dessas atividades seria determinante para o êxito da implantação do sistema de automação e controle.

- Planejamento das atividades;

Cronograma detalhado e que permitisse alterações de acordo com as necessidades da construção;

- Definição dos típicos de configuração;

Buscar o alinhamento entre o padrão da empresa e as melhores práticas do mercado. Não se pode começar uma configuração sem determinar o seu padrão.

- Testes para validação do programa aplicativo;

Um teste prévio da lógica de operação, evita perda de muitas horas na fase de comissionamento.

- Testes de Integração entre o sistema de automação e os dispositivos elétricos;

- Controle das horas de campo;

O descontrole nas atividades de campo é a causa principal do aumento do custo do projeto de automação.

- Documentação;

Controle da documentação emitida, atas de reunião e elaboração de relatórios diário de obra, para permitir o rastreamento das informações.

- Comunicação;

Através de um bom plano de comunicação, podemos evitar retrabalhos nas atividades. 
- Treinamento;

Treinamento com escopo bem definido e no momento certo é fundamental para que as atividades sejam executadas com assertividade.

\section{SOLUÇÕES DE ENGENHARIA}

Com a participação do fornecedor na fase inicial da engenharia detalhada foi possível estabelecer, as melhores soluções de engenharia para aplicar no projeto. Importante ressaltar, que no fechamento comercial do pacote MAC para o sistema de automação e controle, foi previsto verba adicional para permitir aditivos no contrato, para os casos de soluções de engenharia não previstas na fase de negociação.

Principais soluções de engenharia:

- Utilização da biblioteca padrão do fornecedor - PCSD, para os típicos de configuração, visando utilizar as melhores práticas do fornecedor e reduzir os riscos na implantação das lógicas de controle;

- Utilização do padrão HCD - Human Centered Design, para a elaboração das telas de operação do sistema de supervisão. Nova concepção de tela, com elementos gráficos mais discretos, redução na aplicação de cores e na densidade das informações;

- Utilização de equipamentos de automação de última geração, aplicando toda a potencialidade de recursos do fornecedor do sistema;

- Utilização de sistema de segurança para estabelecer o controle do sistema de combustão do forno;

- Utilização de instrumentação Wireless para medição de temperatura no forno;

- Integração dos relés de comando de motores no sistema de gerenciamento de ativos;

- Utilização do conceito de terminal de válvulas com aquisição de dados integrada, nos painéis de comando de válvulas;

- Substituição da tecnologia de medição de nível da calha da prensa de rolos de sistema radioativo por extensiômetros;

- Aplicação de medidor tipo Coriolis para medição de densidade de polpa de minério;

- Participação dos fornecedores de equipamentos mecânicos nos testes de Plataforma (TAF) e nos testes de comissionamento.

\section{BENEFÍCIOS DO MAC}

Podemos considerar o modelo de contratação do sistema de automação para o projeto P4P, com um fator decisivo para o sucesso do projeto.

Os ganhos estão evidenciados nos seguintes fatos:

- O sistema de automação foi entregue, totalmente padronizado, incluindo os fornecimentos do tipo turn-key. (Forno e máquinas do pátio).

- O novo padrão de tela, utilizando a filosofia HCD foi aprovado pelos operadores.

- O comissionamento da planta foi realizado de forma tranquila, com um tempo mínimo de operação assistida e a passagem de bastão, para as equipes da rotina foi bastante suave e sem ocorrências críticas. 
- Não tivemos problemas nas redes industriais, estabelecida à comunicação, checada a integridade da rede, não houve interrupções na comunicação dos dados com o sistema de automação.

- O dimensionamento das equipes atendeu integralmente as necessidades da construção e do comissionamento, não havendo necessidade de aumento de efetivo durante o pico da obra. O planejamento da obra permitiu identificar oportunidades de antecipação de serviços, maximização os recursos.

- Os instrumentos fornecidos foram corretamente armazenados e quando necessário, eram localizados sem dificuldade no almoxarifado do projeto.

- $\mathrm{Na}$ fase de engenharia, foi possível reduzir em $30 \%$ a quantidade de controladores.

- Não tivemos atrasos na construção e no comissionamento devido aos serviços executados pela equipe de automação e instrumentação.

- O custo do pacote de automação não sofreu acréscimos por erros de engenharia ou por aumento das horas de campo.

- A redução no número de empresas envolvidas, resultando em maior agilidade na aquisição, entrega e diligenciamento dos equipamentos.

- Assistência de pós venda assegurada.

\section{LIÇÕES APRENDIDAS NO FORNECIMENTO MAC}

Com relação às possibilidades de melhorias, destacamos os seguintes aspectos para o fornecimento tipo MAC:

- Melhorar integração do fornecedor MAC com a empresa projetista;

- Melhorar o fluxo de tramitação de documentos;

- Estabelecer um plano de gestão de mudanças;

- Necessidade da presença de especialistas, durante a fase de montagem de instrumentos mais complexos;

- Considerar no escopo de fornecimento de instrumentos, todos os acessórios para a instalação;

- Dificuldade em controlar o avanço físico do fornecimento, em função da grande quantidade de atividades previstas no escopo.

\section{SISTEMA DE AUTOMAÇÃO EM NÚMEROS}

Temos os seguintes números, para ilustrar a dimensão do projeto de automação, distribuídos entre os sites de Germano e Ubu: 


\begin{tabular}{|r|c|c|}
\hline Descrição & Site Ubu & $\begin{array}{c}\text { Site } \\
\text { Germano }\end{array}$ \\
\hline Estações de Operação & 15 & 23 \\
Servidores & 10 & 18 \\
\hline Licenças de configuração & 4556 & 7 \\
\hline Telas de operação / engenharia & 139 & 5903 \\
\hline Instrumentos inteligentes em FF & 773 & 92 \\
\hline Instrumentos Wireless & 120 & 709 \\
\hline Motores & 730 & 503 \\
\hline Nós - Profibus DP & 1145 & 820 \\
\hline Pontos Discretos & 8600 & 6020 \\
\hline
\end{tabular}

\section{CUSTO DO FORNECIMENTO MAC}

Para fornecimento dos equipamentos, instrumentos e a realização dos serviços previstos no escopo de fornecimento do pacote de automação e controle, foi gasto $1,3 \%$ do valor total do empreendimento.

\section{CONCLUSÃO}

Os desafios apresentados no trabalho foram superados, através da participação do fornecedor do sistema de automação na fase inicial da engenharia detalhada, permitindo o desenvolvimento de soluções compatíveis com o projeto.

O grande volume de atividades, que as disciplinas de automação e instrumentação enfrentam na fase final da construção, foi administrado de forma satisfatória, principalmente, através da priorização da montagem das redes de campo, fundamental para a evolução dos trabalhos de testes e comissionamento dos equipamentos.

Outro ponto relevante foi o pequeno número de ajustes na programação, durante a fase de comissionamento, indicando que os testes de plataforma foram efetivos. Quando necessário, os ajustes na programação foram realizados de forma rápida, devido ao nível de padronização alcançado na programação do sistema.

O modelo de contratação para o sistema de automação e controle do P4P, onde foi contratada uma solução completa de automação, resultou num fornecimento dentro do prazo e custo esperado, simplificando o processo de aquisição, reduzindo as interfaces, estabelecendo um sistema de controle confiável, com tecnologia inovadora e com total aprovação de todos os envolvidos.

Os resultados alcançados pela disciplina de automação e controle, aliados ao resultado global do projeto, indicam a assertividade das ações realizadas no desenvolvimento de todo o projeto, incluindo as fases de engenharia, aquisição, construção e comissionamento, deixando claro que o padrão MAC de fornecimento, para sistemas de automação, trouxe benefícios consideráveis para a Samarco, reduzindo riscos na implementação do projeto de automação e controle. 